Laser Chem. 1988, Vol. 9, pp. 339-358

(C) 1988 Harwood Academic Publishers GmbH

Photocopying permitted by license only

Reprints available directly from the Publisher

Printed in the United Kingdom

\title{
A Simultaneous Photofragment Spectroscopy Measurement of Rovibronic and Translational Excitation in the $\mathrm{CH}_{3} \mathrm{I}$ A-Band Photodissociation System
}

\author{
JOHN F. BLACK† and IVAN POWIS $\ddagger$
}

Department of Chemistry, University of Nottingham, Nottingham NG7 2RD, UK

(Received 18 April, 1988)

The methyl iodide A-band photodissociation is investigated using a photofragment time-of-flight technique coupled with state specific detection of either the iodine atom or methyl radical dissociation products by multiphoton ionization. The method is quite general and allows the fragments' velocity and spatial distributions to be determined simultaneously with their rotational, vibrational and electronic excitation. It is shown that methyl product from two competing dissociation channels, one direct, the other necessitating an initial surface crossing, can be clearly distinguished by virtue of the characteristic translational excitation in each. The different rotational excitation which is observed in these two channels is discussed in terms of the dissociation dynamics in the methyl iodide A-band continuum.

KEY WORDS: Photodissociation, multiphoton ionization, photofragment spectroscopy.

\section{INTRODUCTION}

Molecular photodissociation studies have proved fertile ground for

$\dagger$ Present address: Department of Chemistry, Stanford University, Stanford, Ca 94305, USA.

$\ddagger$ Author for correspondence. 
those seeking to develop an understanding of fundamental chemical reaction dynamics. ${ }^{1,2}$ Preparation of well characterised reactant species is central to this state of affairs and, while such technology as jet cooling is now routinely available, much ingenuity continues to be expended in this area. A choice of method for probing the nascent product species, their quantum state and spatial distributions will, however, be at least as critical for the scope and potential of an experiment.

Two broad approaches to the analysis of product distributions can be identified. The classic photolysis mapping experiments of Bersohn ${ }^{3}$ graphically demonstrated the spatial anisotropy of photodissociation processes through the non-uniform deposition of product species on the surface of a reaction vessel. With the recognition of the theoretical significance of this anisotropy ${ }^{4,5}$ came the further development of so-called photofragment translational spectrometry, in which the fragment spatial distribution is quantitatively mapped out as a function of angle. ${ }^{6-8}$ Moreover, measurement of the products' time-of-flight as they travel from source to detector establishes the velocity, and hence translational energy, distribution in the dissociation. ${ }^{8-13}$ Product selectivity in these machines has traditionally been achieved by a "universal" mass spectrometer detector. ${ }^{9}$ Though totally general in its application, this reveals nothing about the internal excitation of the fragments other than what can be inferred from their translational distribution.

An alternative class of experiment uses some form of optical spectroscopy to probe the product species. ${ }^{1}$ Laser Induced Fluorescence ${ }^{14}$ (LIF) in particular has been of immense importance in this regard, allowing all manner of internal state populations to be established, while the fluorescence polarization indicates details of the product rotational alignment. ${ }^{2,15}$ Recently, Doppler profile measurements which are sensitive to the projection of the fragment velocity onto the optical viewing axis have become prominent. Under suitable conditions they permit the spatial anisotropy to be investigated, ${ }^{16-18}$ as is done in photofragment time-of-flight (TOF) experiments. One of the most significant new developments brought about by sub-Doppler LIF probing, however, is the ability fully to explore such new facets as the vector correlations between the product rotation and separation motions and the initial transition moment. ${ }^{17-21}$

Offsetting the power of these latest LIF Doppler techniques are the 
somewhat restrictive circumstances required for their achievement. Aside from technical and instrumental demands, relatively fast moving fragments having well resolved, uncongested spectral features are an essential prerequisite. There is also the inherent LIF requirement for a fluorescent state which has to be satisfied. Together, these considerations seem likely to restrict such examinations to lighter atomic and diatomic fragments.

In general, therefore, the photofragment time-of-flight spectroscopy experiment and laser spectroscopic product probing seem to offer complementary abilities, the one revealing translational and spatial distributions, the other internal excitation and possible rotational alignment. A combination of the two types of experiment would promise general, or at least wider, applicability and the ability simultaneously to determine the distribution of both internal and external modes of excitation. To bring about this marriage requires that the product's optical transition be somehow detected in coincidence with its time-of-flight (velocity). This coincidence is, of course, implicit in the Doppler profile measurements. One such experiment by Wittig $^{22}$ (on the LIF of CN fragment from ICN photodissociation) serves to reveal the wider potential of a simultaneous velocity and spectroscopy measurement: in this example the different Doppler components of the $\mathrm{CN}$ spectra could be ascribed to the different $\mathrm{CN}$ translational excitation accompanying $I^{2} P_{3 / 2}$ and $I^{* 2} P_{1 / 2}$ formation. The diatomic rotational excitation in two chemically indistinguishable, but dynamically distinct channels can thus be determined.

In this paper we present analogous results for a polyatomic dissociation system, describing how high resolution optical probing is combined with photofragment time-of-flight measurements as foreseen in the preceding paragraph. Laser multiphoton ionization (MPI) spectroscopy is by now well established as an alternative technique to LIF, with some distinct advantages such as its applicability to "dark" non-fluorescent states ${ }^{23,24}$ and it has previously been applied to examine nascent photofragment distributions. ${ }^{25-27}$ Spectroscopic information comes from a resonant multiphoton absorption to a high-lying neutral state; the resonantly excited species is "tagged" or identified by the charge it carries following its subsequent non-resonant photoionization. Detection of the resonant transition is achieved by detecting the charge; however, this is inevitably delayed by the transit time of the ion to a detector and this permits the simultaneous determination of 
flight-time and resonant photon absorption in a suitably designed experiment, just as we require.

From the perspective of a photofragment time-of-flight experiment there are further advantages of MPI detection. First, the ionization can take place immediately at source in a spatially and temporally well defined manner. This reduces uncertainty in the effective ion path length and flight-time compared to experiments which use a spatially extended electron impact ionizer and it is also more efficient on geometrical grounds than ionization immediately prior to the detector. ${ }^{28}$ Second, it offers both species- and state-specific detection of photofragments. Third, following ionization at source the "tagged" ion population can be manipulated by electric fields rather than drifting freely towards the detector. Many variants of the time-of-flight technique are then possible ${ }^{21,29-31}$ as well as electrostatic energy analysis by retarding and/or deflecting field devices. Further, the ability to accelerate the "tagged" species, giving them a known velocity component transverse to the molecular beam inlet flow direction eliminates some of the experimental complexity which is associated with operation at the kinematically constrained optimum source-detector geometry: ${ }^{9,10,13}$ for any $\mathrm{CM}$ release the $\mathrm{LAB}$ frame scattering angle can now always be swung toward a fixed detector by the simple expedient of an applied electric field.

In the following sections we describe our apparatus and then indicate how the experiment performs using the A-band photodissociation of $\mathrm{CH}_{3} \mathrm{I}$ as an example. Finally, we present new rotationally resolved spectra of the methyl fragment emerging from specific competitive dissociation channels.

\section{APPARATUS}

A schematic diagram of the new MPI/TOF apparatus is presented in Figure 1. The ionization region is defined by the intersection of a focussed laser beam and a molecular beam sample inlet. This can be either an effusive jet from a stainless steel needle (i.d. $0.2 \mathrm{~mm}$ ) or an unskimmed pulsed supersonic nozzle expansion. Although only the latter provides cooling of rotational and vibrational modes, each inlet generates substantial translational cooling in a plane normal to the flow direction. ${ }^{30}$ Ions generated in this source can either drift or, by 


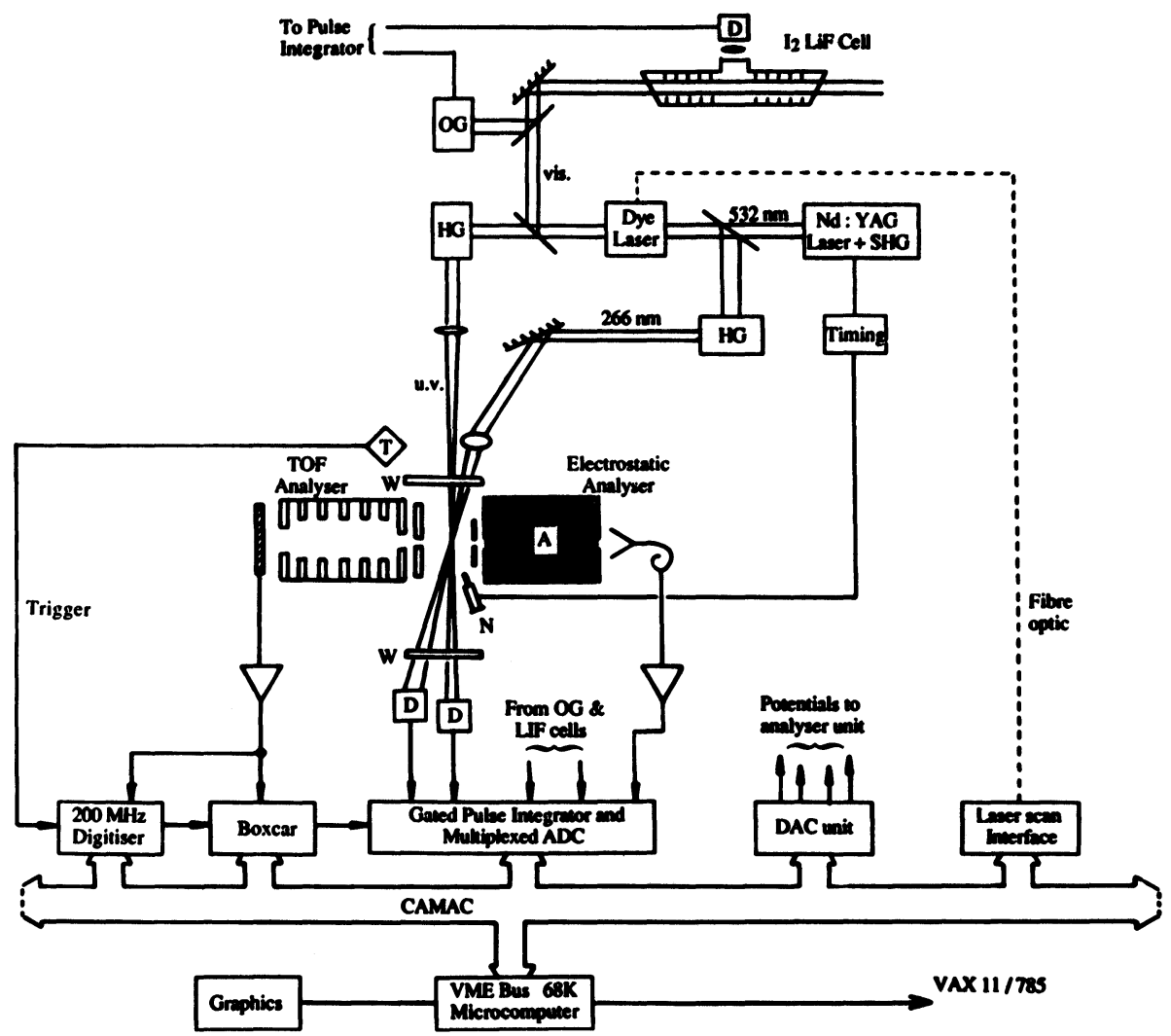

Figure 1 Schematic diagram of the photofragment MPI/TOF apparatus: HG - laser light harmonic generation crystals; OG - optogalvanic cell; $\mathrm{W}$ - vacuum chamber windows; $\mathrm{D}$ - power monitor photodiodes; $\mathrm{T}$ - fast trigger photodiode; $\mathrm{N}$ - pulsed supersonic nozzle or effusive jet (needle) sample inlet; other components as labelled on the diagram.

application of suitable potentials, be accelerated towards one of two analyser units, both of which lie on an axis which is perpendicular to the laser and inlet beams. The first is a $30^{\circ}$ parallel plate electrostatic energy analyser. ${ }^{32}$ The alternative, which is used exclusively for the work reported here, is a small time-of-flight mass/energy analyser. This is constructed as a stack of electrodes so that it can be flexibly operated in either single or twin acceleration field modes by application of appropriate potentials along its length. ${ }^{33}$ The total source- 
detector distance is $6 \mathrm{~cm}$. Surrounding the whole source region is a liquid nitrogen cooled copper panel for additional pumping of residual gases in the vacuum chamber. The base pressure is of the order of $5 \times$ $10^{-8}$ Torr, rising to $\sim 10^{-6}$ Torr with sample admitted.

The ion TOF detector signal is linearly amplified (LeCroy VV100B) and fed to a $200 \mathrm{MHz}$ waveform digitiser (LeCroy TR8828) and a boxcar gated integrator (SRS 250). At very low ion count rates a pulse counting mode is used to record the flight time distributions. For these instances a preamplifier/discriminator (LeCroy MVL100) feeding a multi-hit ns time to digital converter (LeCroy TDC 4208) can be used instead of the waveform digitiser. In all cases a timing trigger signal is obtained from a fast photodiode which picks up scattered laser light. The data acquisition system is CAMAC based and also includes a serial fibre optic interface to the dye laser scan control. The optical transmission of both timing and control signals achieves full electrical isolation between the laser and data system with consequent benefits in the low level signal noise. The CAMAC crate is interfaced to a VME bus computer system for signal averaging, control, etc. and this in turn is linked to mainframe systems for further processing.

For the experiments reported here linearly polarised tunable u.v. radiation was generated by a frequency doubled YAG pumped dye laser system (Quantel Datachrom 5000: R6G dye, $0.1 \mathrm{~cm}^{-1}$ linewidth, 5 ns pulses) and could be attenuated to give pulse energies as desired. A double Fresnel rhomb was used to rotate the laser polarisation. Separation of the u.v. output from the residual dye fundamental was achieved by an arrangement of four Pellin-Broca prisms such that no deviation of the u.v. beam direction occurs as the wavelength is scanned. We then use a portion of the separated visible light for dye laser frequency calibration. Initially this was performed by observing optogalvanic lines in a hollow cathode lamp, then during a wavelength scan accurate frequency calibration is maintained by passing the visible beam through a cell of $I_{2}$ vapour and monitoring the $I_{2} \mathrm{~B} \rightarrow X$ LIF signal simultaneously with the sample MPI signals. The apparent frequency of the former can then be compared with an atlas of the $I_{2}$ spectrum, ${ }^{34}$ allowing results for the latter to be quoted to $\pm 0.1 \mathrm{~cm}^{-1}$.

The doubled dye laser output is focussed through the apparatus with either a $30 \mathrm{~cm}$ cylindrical or spherical lens. Although the $3 \mathrm{rd}$ and 4 th YAG harmonics may also be focussed into the chamber as photolysis sources, this was not required for these experiments, as explained 
below. The exiting laser beam impinges on a pulse energy monitoring photodiode which allows the laser pulse energy to be monitored on a shot to shot basis. For absolute power measurements a calibrated calorimeter power meter can also be inserted in the beam.

\section{PHOTOFRAGMENT TIME-OF-FLIGHT EXPERIMENTS: OPERATION AND PERFORMANCE}

The methyl iodide A-band absorption is a broad continuum feature lying between 200 and $300 \mathrm{~nm}$. Excitation of this continuum leads to an extremely rapid dissociation ${ }^{35}$ to both ground $I^{2} P_{3 / 2}$ and spin-orbit excited $I^{* 2} P_{1 / 2}$ products. ${ }^{8,11-13,36-39}$ At sufficiently high laser intensities the photolysis beam can subsequently multiphoton ionize the photofragments if it is tuned within the A-band to match a two-photon resonance of the fragments..$^{30,40,41}$ A single colour laser will thus photolyse and probe the fragment distribution.

When the laser is tuned to known $I$ atom two-photon resonant ionization frequencies within the A-band range $I^{+}$ions will be generated: ${ }^{30,40}$

$$
\begin{aligned}
& \mathrm{CH}_{3} \mathrm{I} \stackrel{h v_{1}}{\longrightarrow} \mathrm{CH}_{3}+I^{* 2} P_{3 / 2} \stackrel{(2+1) h v_{1}}{\longrightarrow} I^{+} \\
& \left.\mathrm{CH}_{3} \mathrm{I} \stackrel{h v_{1}}{\longrightarrow} \mathrm{CH}_{3}{ }^{*}\right)+I^{2} P_{3 / 2} \stackrel{(2+1) h v_{1}}{\longrightarrow} I^{+}
\end{aligned}
$$

If these ions are allowed to drift in a zero field environment toward the ion detector then a TOF spectrum such as shown in Figure 2a is obtained. This is essentially the same experiment and result as in previous photofragment investigations of this system ${ }^{11-13}$ except that, because the ionization step is state-selective, only a single broad peak corresponding to one of the two dissociation channels is observed-in Figure 2 it is the $I^{*}$ product from (1) which we detect. If instead the laser is tuned to an adjacent resonant ionization of the ground state $I$ atom, detecting channel (2), then a qualitatively similar peak is obtained, shifted to shorter flight times by virtue of the greater kinetic energy available in this channel.

Figure $2 \mathrm{~b}$ contains the result of using a rather different mode of operation. In this case the ions, after drifting free for $0.5 \mathrm{~cm}$, are accelerated by a field of $40 \mathrm{~V} \mathrm{~cm}-1$ over a distance of $1.5 \mathrm{~cm}$ and then once again drift in a field free region of $4 \mathrm{~cm}$ to the detector. This 

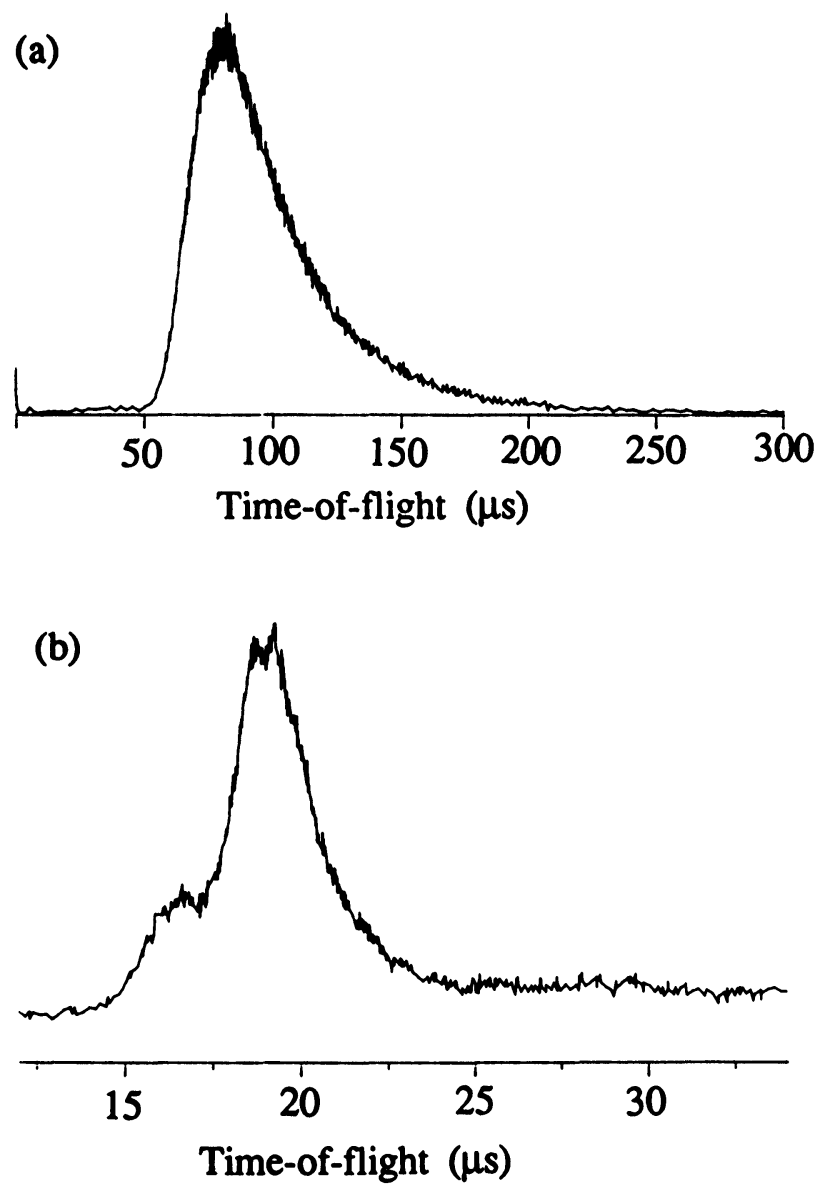

Figure 2 Time-of-Flight peaks of $I^{* 2} P_{1 / 2}$ photofragment, resonantly ionized by $(2+1)$ $281.73 \mathrm{~nm}$ photons. (a) Zero source extraction field, zero acceleration field, $80 \mathrm{~ns}$ resolution. 50,000 laser shots averaged; (b) Zero source extraction field, $40 \mathrm{~V} \mathrm{~cm}^{-1}$ acceleration field, $10 \mathrm{~ns}$ resolution. 5000 laser shots averaged. See text for further details.

unconventional use of the TOF spectrometer is primarily designed to help reveal structure in the underlying velocity distribution of the fragments. The shoulder on the short flight-time side of the peak in Figure $2 \mathrm{~b}$ can be attributed, with the aid of computer simulations, to the $\mathrm{CH}_{3}\left(v_{2}=0\right)$ feature in the $\mathrm{CM}$ translational energy distribution, while the main peak comprises the remaining $\mathrm{CH}_{3}\left(v_{2}=1,2, \ldots\right)$ 
components. Of course both the smooth and partially structured arrival time distributions in Figure 2 should transform to a single given velocity distribution and there are sound reasons for attempting to infer the methyl $v_{2}$ distribution from this. ${ }^{11-13,38}$ However, because of the assumptions which are required to do so it is reassuring to be able to see visible structure corresponding to the $v_{2}$ quantum in the raw data as here.

One drawback to laser ionization in time-of-flight experiments intended to measure kinetic energy is the high ion density created in the small focal volume. This can generate a Coulomb "explosion" due to mutual space charge repulsion of the ions. It is readily shown from simple electrostatic considerations that as the number of ions in the focal volume of the laser (say $\sim 100 \mu$ diameter) increases, those ions at the periphery experience repulsive forces which can generate the equivalent of several hundred meV extra translational energy. For heavy ions, such as $I^{+}$, which only slowly drift from the focal volume this can become a quite serious problem. It is particularly acute in the zero source field experiments of Figure 2. Here the collection efficiency at the detector is low, and the low count rates require operation in a pulse counting mode, which unfortunately makes it difficult to detect the onset of space-charge induced flight-time distortion.

To minimise these problems while still maintaining a useful count rate we have used cylindrical lenses giving a soft, extended focus along the molecular beam direction for all the $I$ atom TOF experiments. Software has also been written which continuously monitors the laser pulse energy and excludes the more energetic pulses from the TOF averaging which is performed. Because of the non-linearity of the MPI process the natural shot-shot laser power fluctuations can be responsible for a substantial variation in the number of ions produced per pulse and hence the extent of Coulomb broadening of the TOF peak, even when operating at a constant low mean power.

A preferable mode of operation, however, is to use a field to accelerate ions from the source. For given ionization conditions this has the effect of sweeping up far more ions, even those whose initial velocity is away from the detector, resulting in a much improved detector count rate. Figure 3 shows the results of such operation and the improved collection efficiency is seen in the signal to noise ratio achieved after averaging 5000 shots as compared to the zero field experiment for which 50,000 shots were averaged. Because of the 

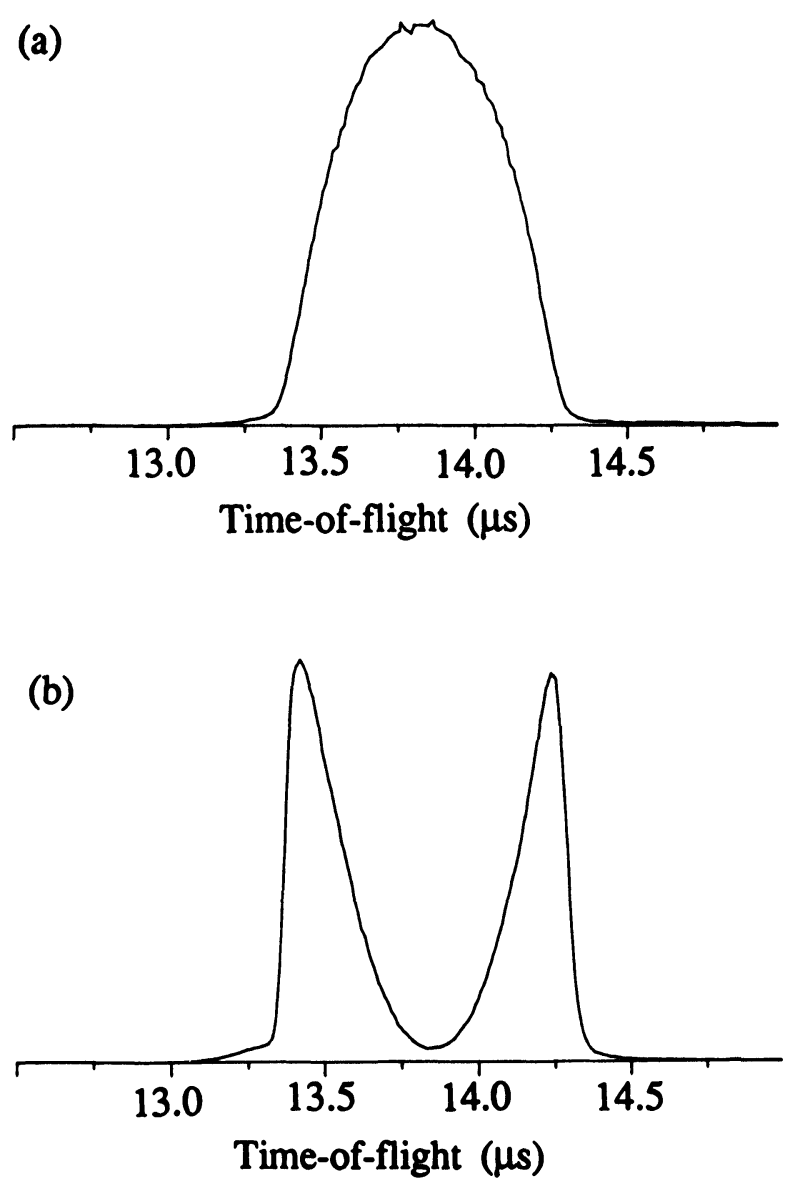

Figure 3 Time-of-Flight peaks of $I^{* 2} P_{1 / 2}$ photofragment, resonantly ionized by $(2+1)$ $280.99 \mathrm{~nm}$ photons and recorded using a single extraction/acceleration field of $12 \mathrm{~V} \mathrm{~cm}$ and $5 \mathrm{~ns}$ resolution. 5000 laser shots averaged. Photolysis laser polarisation, $\varepsilon_{\mathrm{p}}$ set perpendicular (a) and parallel (b) to the instrumental flight axis.

improved signal the TOF peak can now be viewed in real time and the symmetric broadening of space charge repulsion is readily perceived and hence avoided.

Although accelerating fields impose an extra component of velocity onto the ion trajectories, the initial velocity distribution of the ion is still mapped onto the flight time distribution in a well understood 
manner; so too is the initial angular (spatial) distribution. This latter is seen in the difference between Figures $3 a$ and $3 b$ which differ only in the orientation of the laser polarisation vector, $\varepsilon_{\mathbf{p}}$, relative to the instrumental axis. The pronounced splitting of the peak shape when the laser polarisation is set parallel to the flight axis is a clear indication that fragments are preferentially scattered parallel to the $\varepsilon_{\mathbf{p}}$ vector; forward scattered particles arrive early, backward scattered particles, late.

The velocity and angular distributions have been deconvoluted from TOF peaks such as those of Figure 3 allowing the anisotropy and the $v_{2}$ vibrational distribution inferred from the $\mathrm{CM}$ energy release to be determined. ${ }^{30}$ There are distinct differences between the two channels in both distributions and this can be interpreted in terms of dissociation over two excited surfaces. While $I^{*}$ formation is apparently a direct dissociation process over a single surface, $\langle\beta\rangle=1.95$, the results for the $I$ photofragment, $\langle\beta\rangle=1.75$, are consistent with a non-adiabatic interaction inducing crossing to the second surface.

\section{ROTATIONAL EXCITATION OF THE METHYL PHOTOFRAGMENT}

\section{Results}

Just as the $I$ atom photofragment can be ionized and detected by exciting a suitable multiphoton resonance lying within the one photon region of the parent A-band, so too can the methyl fragment. Figure 4 shows the methyl TOF peaks obtained by exciting in the centre of the methyl two-photon $\tilde{X}^{2} A_{2}^{\prime \prime} \rightarrow 4 p^{2} A_{2}^{\prime \prime}$ transition at $286.3 \mathrm{~nm} .{ }^{41-43}$ Once again, with the laser $\varepsilon_{\mathbf{p}}$ vector parallel to the axis the TOF peak is split into forward- and back-scattered components under various conditions of source extraction field. It is also clear that there are two contributory sources of methyl, one travelling faster than the other and so giving increased splitting as the forward-scattered ions arrive earlier and the backward, which now take even longer to decelerate and reverse direction in the source, arrive later. Unlike the previous $I$ atom TOF peaks, for which there was an accompanying distribution of methyl internal excitations and hence a range of possible translational energies, here the energetics are much more precisely determined because the methyl rovibronic state is selected by the chosen resonant 

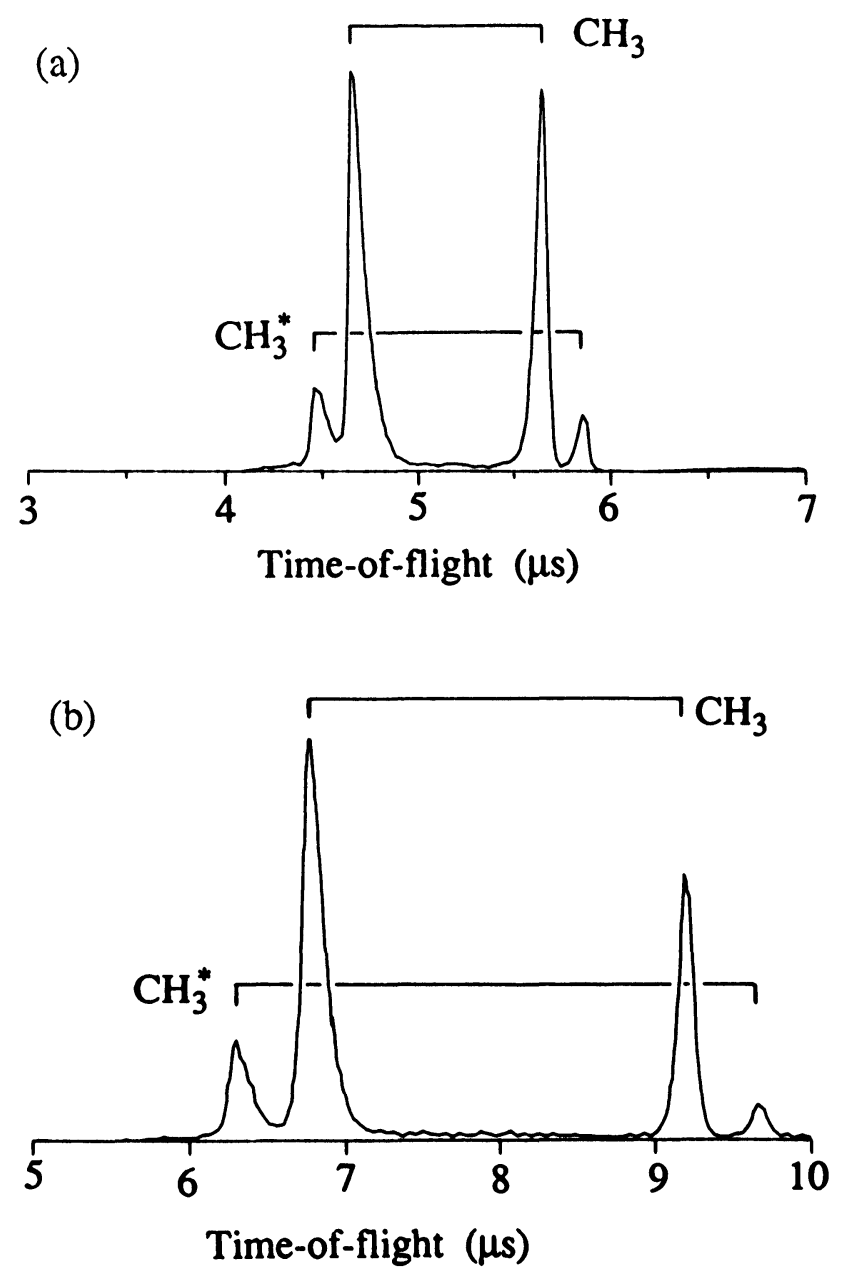

Figure 4 Time-of-Flight peaks of resonantly ionized $\left(286.3 \mathrm{~nm}\right.$ photons) $\mathrm{CH}_{3}(v=0)$ photofragments. Two contributing channels, $\mathrm{CH}_{3}+I^{*}$ and $\mathrm{CH}_{3}\left({ }^{*}\right)+I$ are identified. Laser polarisation set parallel to the instrumental flight axis, 5000 shots averaged, $5 \mathrm{~ns}$ resolution. A single extraction/acceleration field of either (a) $10 \mathrm{~V} \mathrm{~cm}^{-1}$ or (b) $4.0 \mathrm{~V} \mathrm{~cm}-1$ (lower) was used. 
excitation wavelength. The slower source of methyl is then readily identified with the $I^{*}$ dissociation channel, (1), while the faster, which we denote $\mathrm{CH}_{3}{ }^{\left({ }^{*}\right)}$ for convenience, is the product from the ground state $I$ atom channel, (2). The polyatomic fragments emerging from two different channels are thus distinguished by the different kinetic energies they carry.

Channel resolved methyl excitation spectra can be obtained by integrating the $\mathrm{CH}_{3}$ or $\mathrm{CH}_{3}{ }^{(*)}$ peak intensities as the laser is scanned. In practice we used the back scattered components of the TOF peak for this purpose. Both an effusive jet and a supersonic nozzle expansion have been used for the sample inlet, but better results were obtained with the jet source. One also has greater confidence that no complicating dimer formation occurs with the effusive inlet and this was therefore employed for the spectra presented below.

A spherical $f=30 \mathrm{~cm}$ lens was used to focus the laser for these experiments and we determined that the $300 \mu \mathrm{J}$ pulse energies used were insufficient to perturb the spectra through power broadening/ saturation effects. ${ }^{43}$ Equally important there was no Coulombic broadening of the TOF peak shapes which might allow the slower $\mathrm{CH}_{3}$ component to spread into the $\mathrm{CH}_{3}{ }^{\left({ }^{*}\right)}$ component with consequent contamination of the channel resolved spectra. After recording, the spectra were normalised by division by the square of the laser power to compensate for power variations experienced while scanning across the dye tuning curve. This is a sufficient and appropriate correction as the initial one-photon dissociation step is evidently saturated and the fragment signal shows a quadratic power dependence under these conditions. ${ }^{41,43}$

The $(2+1)$ MPI spectra of the methyl $4 p_{z} 0-0$ origin band are shown in Figure 5. In these plots the very intense central $Q$ branch has been truncated to allow the $O, P, R, S$ branch structure to be more clearly seen and calculated positions of the $O, P, R, S^{43}$ branch structure are marked on the upper trace. The $\mathrm{CH}_{3}{ }^{\left({ }^{*}\right)}$ yield is the lesser of the two possible dissociation channels and so gives a weaker signal. Despite this the differences in rotational structure for these two $v=0$ dissociation channels are pronounced and obvious.

It is known that a distribution of methyl $v_{2}$ vibrational excitation is produced in this photodissociation ${ }^{37,38}$ and so hot bands are also expected in the MPI spectrum. ${ }^{29,31}$ Figure 6 contains the $4 p_{\mathrm{z}} 2{ }_{1}^{1}$ band spectra for $\mathrm{CH}_{3}$ and $\mathrm{CH}_{3}{ }^{(*)}$. Because of the uncertainties created by 


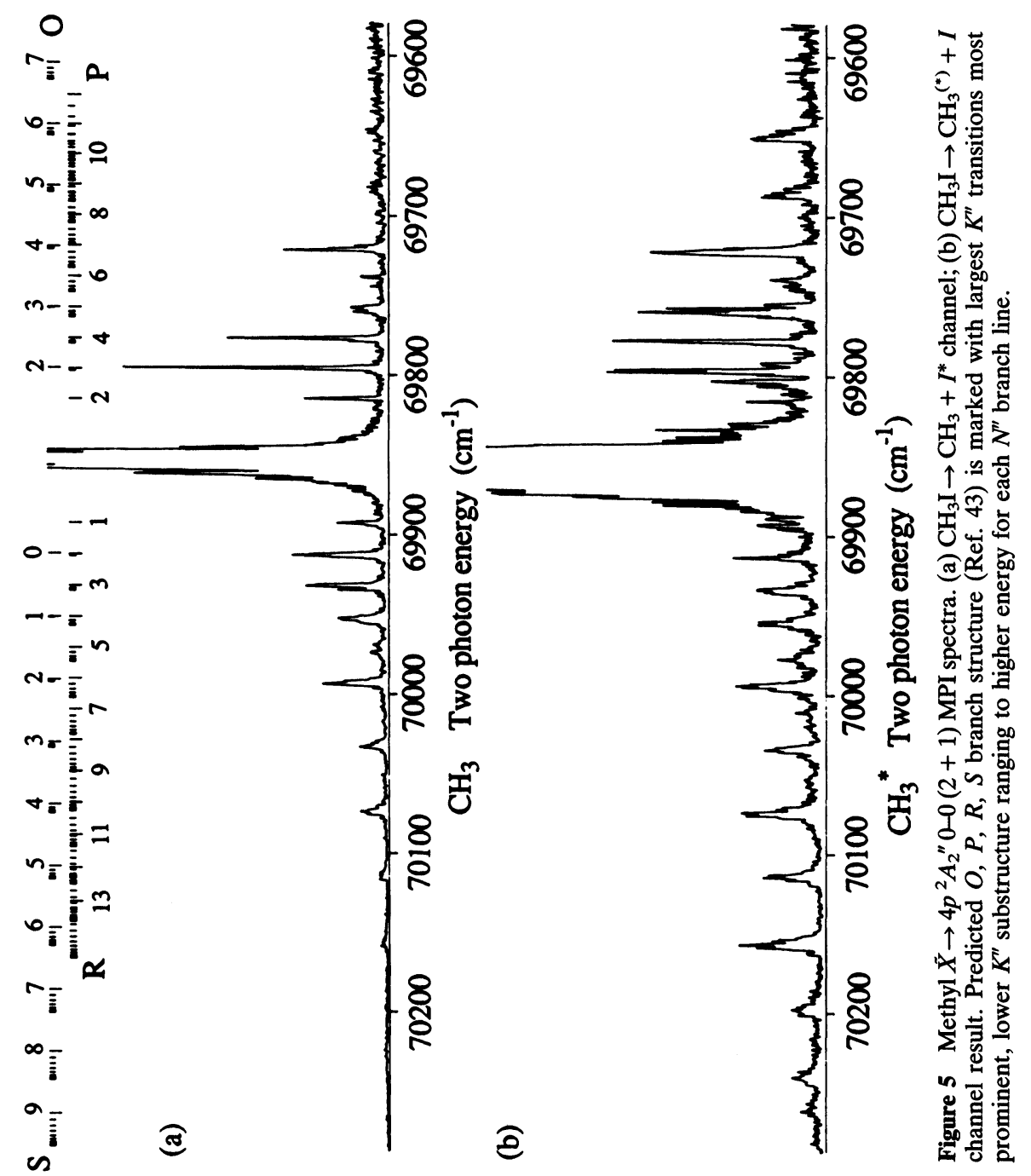




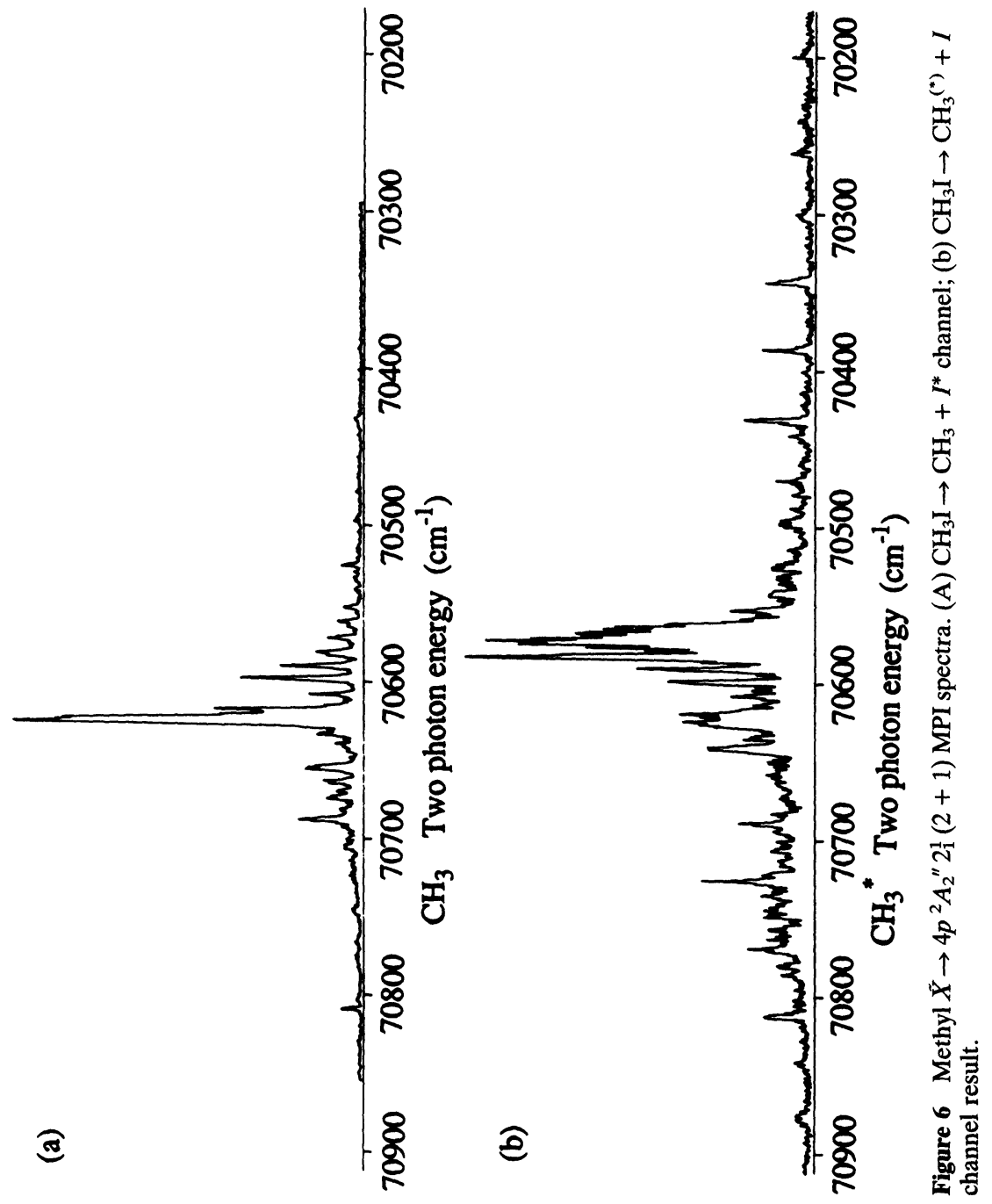


the unknown initial population, rotational alignment, and competing predissociation of the intermediate methyl Rydberg state ${ }^{43}$ a full rotational analysis of this spectrum is not yet available. Nevertheless, distinct $Q$ branch features and, for the $\mathrm{CH}_{3}{ }^{(*)}$ channel, $O$ and $S$ branch lines are visible. Again there are marked differences between the two channels indicating the very different nascent rotational populations created by these different dissociation pathways.

\section{Discussion}

The A-band excitation of methyl iodide predominantly populates two repulsive states. ${ }^{44,45}$ As shown in Figure 7 one, the ${ }^{3} Q_{0}$ surface, correlates with $\mathrm{CH}_{3}+I^{*}$, while the alternative ${ }^{1} Q$ state correlates asymptotically with $\mathrm{CH}_{3}{ }^{(*)}+I$. In fact at the particular photolysis wavelengths of this study the oscillator strength is carried almost exclusively by the transition to the former ${ }^{3} Q_{0}$ surface ${ }^{45}$ so that we observe the $\mathrm{CH}_{3}$ channel as a direct dissociation, and the $\mathrm{CH}_{3}{ }^{(*)}$ channel as a predissociation brought about by an electronically nonadiabatic interaction between the ${ }^{3} Q_{0}$ and ${ }^{1} Q$ surfaces.

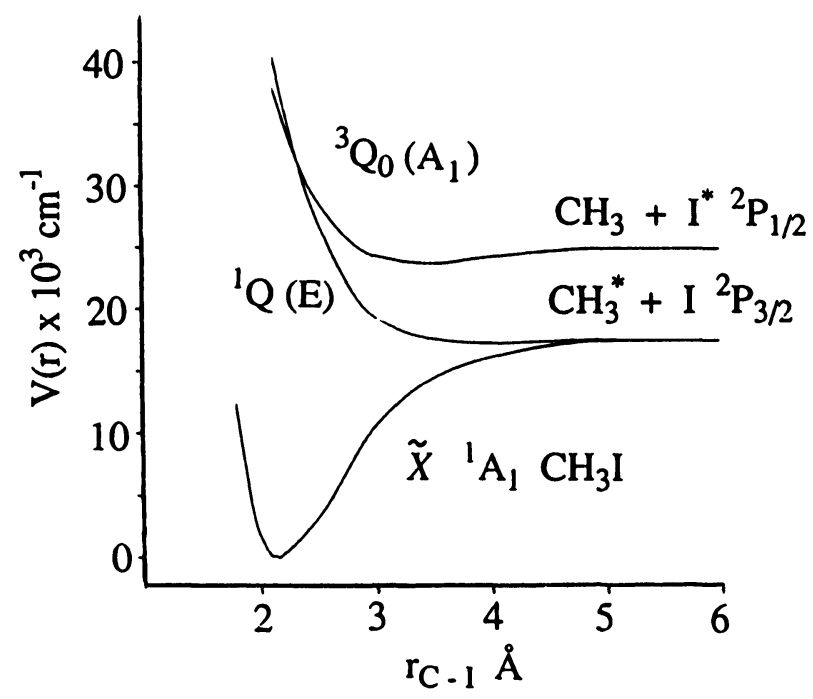

Figure 7 Ground state $\left(A_{1}\right)$ and excited state ${ }^{3} Q_{0}\left(A_{1}\right)$ and ${ }^{1} Q(E)$ potentials for methyl iodide (Ref. 46). 
From a qualitative examination of the $O, P, R, S$ structure of Figure $5 a$ it can be seen that $\mathrm{CH}_{3}(v=0)$ rotational levels for $N^{\prime \prime}$ up to 6 are populated. Elsewhere ${ }^{43}$ we have shown that, ignoring the influence of rotational alignment on the observed transition strength and making some allowance for the heterogeneous predissociation of the $4 p$ Rydberg levels, this roughly corresponds to a rotational temperature of $200 \mathrm{~K}$. This apparent cooling (compared to the $300 \mathrm{~K}$ parent rotational temperature) can be predicted by a Franck-Condon model in which exit channel interactions do not influence the rotational distribution. Thus, the initial parent rotational angular momentum is simply correlated with that of the polyatomic methyl fragment: rotation about the parent top axis passes directly into fragment top axis rotation but, to conserve angular momentum, much of the perpendicular rotation passes into orbiting motion of the heavy $I^{*}$ fragment, leaving the methyl fragment to rotate mainly around its symmetry axis. It follows that those levels for which $K^{\prime \prime} \simeq N^{\prime \prime}$ should be preferentially populated. The lack of any exit channel effects is entirely plausible for a direct dissociation over the repulsive ${ }^{3} Q_{0}$ surface in which the three-fold $C_{3}$ symmetry axis is conserved.

In contrast, exit channel interactions do seem to be important in accounting for the $\mathrm{CH}_{3}{ }^{(*)}$ channel rotational structure. A qualitative examination of Figure $5 \mathrm{~b}$ reveals that rotational levels for up to $N^{\prime \prime}=8$ are now observed. It is unlikely that different rotational alignments could alone account for the apparent differences between the $\mathrm{CH}_{3}$ and $\mathrm{CH}_{3}{ }^{\left({ }^{*}\right)}$ spectra in Figure 5, since such effects would primarily be expected to influence the relative intensities of the $(O, S)$ and $(P, R)$ branch lines of a given $N^{\prime \prime}$ rather than adjacent lines of a given branch. ${ }^{47}$ Almost certainly what is observed here is more extensive rotational excitation in the $\mathrm{CH}_{3}{ }^{(*)}(v=0)$ dissociation channel. The same conclusion is arrived at regarding the $\mathrm{CH}_{3}$ and $\mathrm{CH}_{3}{ }^{(*)}(v=1)$ channels following an examination of Figure 6; indeed, here the rotational population differences may be even more pronounced.

The $\mathrm{CH}_{3}{ }^{(*)}$ channel is dynamically more complex than the $\mathrm{CH}_{3}$ channel, ${ }^{12,13,29,30}$ as it involves a surface crossing between the ${ }^{3} Q_{0}$ and ${ }^{1} Q$ surfaces. In the $C_{3 \mathrm{v}}$ point group these are of $A_{1}$ and $E$ symmetry respectively and can be coupled by a vibronic interaction. It has been argued $^{13}$ that this is most likely brought about by the parent $v_{6} e$ type wagging mode and it was further predicted that this would allow extra energy to pass into rotation in the $\mathrm{CH}_{3}{ }^{\left({ }^{*}\right)}$ channel, just as is observed 
here. Alternatively and somewhat equivalently, it can be noted that the ${ }^{1} Q(E)$ surface is electronically degenerate so that the system will experience a Jahn-Teller distortion in the uncoupling region following the surface crossing. Hence, the simple $C_{3}$ axis reaction coordinate of the direct ${ }^{3} Q_{0}$ dissociation will no longer obtain and the transition state will be bent, thus allowing an extra torque and consequent rotational excitation to be imparted to the methyl group.

There is another possible coupling mechanism, due to electronicrotation Coriolis interaction. In the situation considered here parent molecule $R_{x}, R_{y}$ axis rotations (i.e. those perpendicular to the $C_{3}$ symmetry axis) could conceivably couple the $A_{1}$ and $E$ symmetry electronic states. This suggests that the fragment methyl resulting from the $\mathrm{CH}_{3}{ }^{\left({ }^{*}\right)}$ channel might have a greater propensity for perpendicular rotation when compared to the direct $\mathrm{CH}_{3}$ dissociation product because of the correlation between parent and fragment rotational angular momentum. Put another way, for a given $N^{\prime \prime}$ rotational quantum number lower associated $K^{\prime \prime}$ levels can be expected in the predissociation channel. In spectra such as those here transitions from lower $K^{\prime \prime}$ levels will range to higher energy, as marked on Figure 5. Although there is no fully resolved $K$-substructure in the spectra of Figure 5 the higher members of the $\mathrm{CH}_{3}{ }^{(*)} \mathrm{O}$ and $\mathrm{S}$ branches do shade to the red and indicate that low $K^{\prime \prime}$ levels are populated. We can also draw attention to the $Q$ branches in Figure 5. In the $\mathrm{CH}_{3}{ }^{\left({ }^{*}\right)}$ case there is considerable broadening of this feature reflecting the greater rotational excitation, mostly appearing to the blue of the band origin. This is just the region where the (unresolved) transitions from higher $N^{\prime \prime}$, lower $K^{\prime \prime}$ levels are predicted, ${ }^{43}$ whereas higher $N^{\prime \prime}$, higher $K^{\prime \prime}$ transitions should lie to the red of the origin. Without a full model for the rotationally aligned linestrengths it is not possible to assert that there is a definite propensity for the $\mathrm{CH}_{3}{ }^{\left({ }^{*}\right)}$ to have perpendicular rotation, but it certainly cannot be ruled out on the basis of these observations. A further study of the temperature dependence of the rotational excitations would be useful in this regard.

\section{Acknowledgements}

This work was supported by a research grant from the SERC who also awarded a studentship to JFB. Some additional equipment funds were provided by a grant from the Nuffield Foundation. 


\section{References}

1. S. R. Leone, Adv. Chem. Phys. 50, 255 (1982).

2. J. P. Simons, J. Phys. Chem. 88, 1287 (1984).

3. J. Solomon, C. Jonah, P. Chandra and R. Bersohn, J. Chem. Phys. 55, 1908 (1971).

4. R. N. Zare, Mol. Photochem. 4, 1 (1972).

5. S. Yang and R. Bersohn, J. Chem. Phys. 61, 4400 (1974).

6. M. Dzvonik, S. Yang and R. Bersohn, J. Chem. Phys. 61, 4409 (1974).

7. G. E. Busch and K. R. Wilson, J. Chem. Phys. 56, 3626; 3638 (1972).

8. S. J. Riley and K. R. Wilson, Far. Disc. Chem. Soc. 53, 132 (1972).

9. A. M. Wodtke and Y. T. Lee, Molecular Photodissociation Dynamics (eds. M. N. R. Ashfold and J. E. Baggott, Royal Society of Chemistry, London, 1987), Chap. 2, pp. 31-59.

10. L. J. Butler, E. J. Hintsa, S. F. Shane and Y. T. Lee, J. Chem. Phys. 86, 2051 (1987).

11. R. K. Sparks, K. Shobatake, L. R. Carlson and Y. T. Lee, J. Chem. Phys. 75, 3838 (1981).

12. G. N. A. Van Veen, T. Baller, A. E. de Vries and N. J. A. Van Veen, Chem. Phys. 87, 405 (1984).

13. M. D. Barry and P. A. Gorry, Mol. Phys. 52, 461 (1984).

14. J. L. Kinsey, Ann. Rev. Phys. Chem. 28, 349 (1977).

15. C. H. Greene and R. N. Zare, J. Chem. Phys. 78, 6741 (1983); Ann. Rev. Phys. Chem. 33, 119 (1982).

16. R. Schmiedl, H. Dugan, W. Meier and K. H. Welge, Z. Phys. 304A, 137 (1982).

17. M. P. Docker, A. Hodgson and J. P. Simons, Chem. Phys. Lett. 128, 264 (1986); Far. Disc. Chem. Soc. 82, 25 (1986).

18. M. Dubs, U. Bruhlmann and J. R. Huber, J. Chem. Phys. 84, 3106 (1986).

19. J. P. Simons, J. Phys. Chem. 91, 5378 (1987).

20. G. E. Hall, N. Sivakumar, P. L. Houston and I. Burak, Phys. Rev. Lett. 56, 1671 (1986).

21. G. E. Hall, N. Sivakumar, R. Ogorzalek, G. Chawla, H.-P. Haerri and P. L. Houston, Far. Disc. Chem. Soc. 82, 13 (1986).

22. I. Nadler, D. Mahgerefteh, H. Reisler and C. Wittig, J. Chem. Phys. 82, 3885 (1985).

23. P. M. Johnson, Appl. Opt. 19, 3920 (1980).

24. M. N. R. Ashfold, Mol. Phys. 58, 1 (1986).

25. R. J. S. Morrison and E. R. Grant, J. Chem. Phys. 77, 5994 (1982).

26. B. H. Rockney and E. R. Grant, J. Chem. Phys. 79, 708 (1983).

27. N. Goldstein, G. D. Greenblatt and J. R. Weisenfeld, Chem. Phys. Lett. 96, 410 (1983).

28. H. J. Krautwald, L. Schnieder, K. H. Welge and M. N. R. Ashfold, Far. Disc. Chem. Soc. 82, 99 (1986).

29. R. Ogorzalek, G. E. Hall, H.-P. Haerri and P. L. Houston, J. Phys. Chem. 92, 5 (1988).

30. J. F. Black and I. Powis, Chem. Phys., in press.

31. J. W. Thoman Jnr., D. W. Chandler, D. H. Parker and M. H. M. Janssen, Laser Chem., in press.

32. I. Powis, P. I. Mansell and C. J. Danby, Int. J. Mass Spec. Ion Phys. 32, 15 (1979).

33. W. C. Wiley and I. H. McLaren, Rev. Sci. Inst. 26, 1150 (1955).

34. S. Gerstenkorn and P. Luc, Atlas du Spectre d'Absorption de la Molecule d'Iode (C.N.R.S., Paris, 1978).

35. J. L. Knee, L. R. Khundkar and A. H. Zewail, J. Chem. Phys. 83, 1996 (1985). 
36. T. F. Hunter and K. S. Kristjanssen, Chem. Phys. Lett. 58, 291 (1978).

37. S. L. Baughcum and S. R. Leone, J. Chem. Phys. 72, 6531 (1980).

38. H. W. Hermann and S. R. Leone, J. Chem. Phys. 76, 4766 (1982).

39. W. P. Hess, S. J. Kohler, H. K. Haugen and S. R. Leone, J. Chem. Phys. 84, 214: (1986).

40. A. Gedanken, M. B. Robin and Y. Yafet, J. Chem. Phys. 76, 4798 (1982).

41. Y. Jiang, M. R. Giorgi-Arnazzi and R. B. Bernstein, Chem. Phys. 106, 171 (1986).

42. J. W. Hudgens, T. G. DiGiuseppe and M. C. Lin, J. Chem. Phys. 79, 571 (1983).

43. J. F. Black and I. Powis, J. Chem. Phys., in press.

44. D. Porret and C. F. Goodeve, Proc. Roy. Soc. A165, 31 (1938).

45. A. Gedanken and M. D. Rowe, Chem. Phys. Lett. 34, 39 (1975).

46. M. Tadjeddine, J. P. Flament and C. Teichteil, Chem. Phys. 118, 45 (1987).

47. A. C. Kummel, G. O. Sitz and R. N. Zare, J. Chem. Phys. 85, 6874 (1986). 